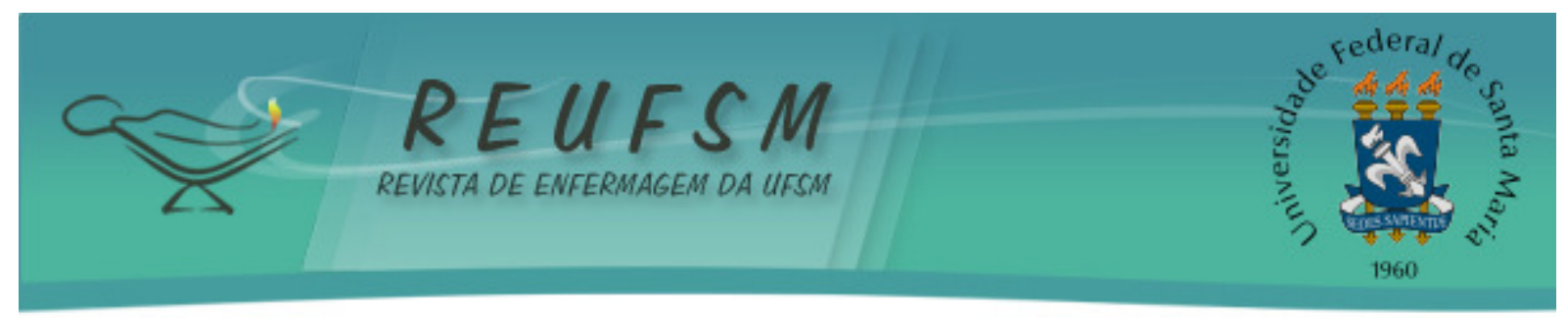

ARTIGO ORIGINAL

\title{
REPRESENTAÇÕES SOCIAIS DO PROCESSO DE ADOECIMENTO DOS PACIENTES ONCOLÓGICOS EM CUIDADOS PALIATIVOS NO DOMICÍLIO
}

\author{
SOCIAL REPRESENTATIONS OF ONCOLOGICAL PATIENTS' ILLNESS PROCESS \\ UNDER PALLIATIVE CARE AT HOME
}

REPRESENTACIONES SOCIALES DEL PROCESO DE ADOLECIMIENTO DE PACIENTES ONCOLÓGICOS EN CUIDADOS PALIATIVOS EN EL DOMICILIO

\author{
Michele Rodrigues Matos ${ }^{1}$ \\ Rosani Manfrin Muniz ${ }^{2}$ \\ Michele Cristiene Nachtigall Barboza ${ }^{3}$ \\ Aline da Costa Viegas ${ }^{4}$ \\ Juliana Amaral Rockembach ${ }^{5}$ \\ Luiz Guilherme Lindemann ${ }^{6}$
}

Doi: $10.5902 / 2179769225801$

RESUMO: Objetivo: conhecer as representações sociais do processo de adoecimento de pacientes oncológicos em cuidados paliativos no contexto domiciliar. Método: estudo exploratório, descritivo com abordagem qualitativa, desenvolvido com dez pacientes oncológicos em cuidados paliativos, no período de maio a julho de 2015 , utilizando-se da análise temática, a qual foi desenvolvida a partir da Teoria das Representações Sociais de Moscovici. Resultados: o processo de adoecimento trouxe consigo perdas, restrições referentes à vida diária e à capacidade laboral e para este enfrentamento a rede de apoio se fez presente na figura da família e da rede social, da espiritualidade e da equipe de saúde. Considerações finais: percebeu-se que adoecer por câncer em cuidados paliativos, pode causar restrições na vida diária e na capacidade produtiva, além de modificar a representação social que associa o câncer à morte, pois independente do estágio em que os pacientes encontram-se, estes apresentaram esperança da cura, associado ou não a fé.

Descritores: Doença crônica; Neoplasias; Cuidados paliativos; Doente terminal.

\footnotetext{
${ }^{1}$ Enfermeira, Mestre em Ciências, Secretaria Estadual de Saúde do Rio Grande do Sul, Pelotas, Rio Grande do Sul, Brasil. E-mail: michele.rodriguesmatos@gmail.com

2 Enfermeira, Doutora em Enfermagem, Faculdade de Enfermagem e Programa de Pós Graduação em Enfermagem da Universidade Federal de Pelotas, Pelotas, Rio Grande do Sul, Brasil. E-mail: romaniz@terra.com.br

3 Enfermeira, Mestre em Enfermagem, Faculdade de Enfermagem e Programa de Pós Graduação em Enfermagem da Universidade Federal de Pelotas, Pelotas, Rio Grande do Sul, Brasil. E-mail: michelenachtigall@yahoo.com.br

${ }^{4}$ Enfermeira, Mestre em Ciências, Hospital Escola da Universidade Federal de Pelotas/EBSERH e Programa de Pós Graduação em Enfermagem da Universidade Federal de Pelotas, Pelotas, Rio Grande do Sul, Brasil. E-mail: alinecviegas@hotmail.com

${ }^{5}$ Enfermeira, Especialista em Unidade de Terapia Intensiva, Prefeitura Municipal de Santa Cruz do Sul e Programa de Pós Graduação em Enfermagem da Universidade Federal de Pelotas, Santa Cruz do Sul, Rio Grande do Sul, Brasil. E-mail: ju.rockembach@ hotmail.com

${ }^{6}$ Acadêmico de Enfermagem, Faculdade de Enfermagem da Universidade Federal de Pelotas, Pelotas, Rio Grande do Sul, Brasil. E-mail: luguilindemann@ @otmail.com
} 


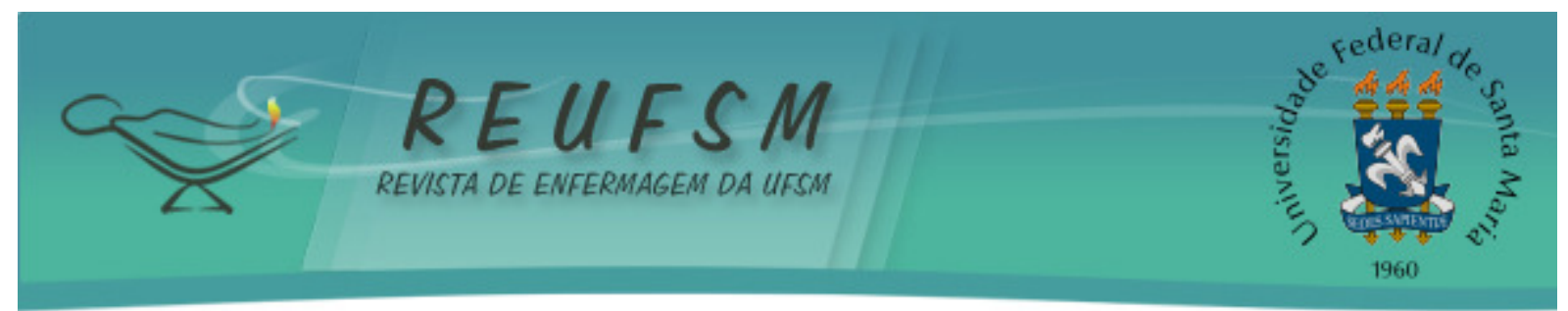

ABSTRACT: Aim: to know social representations of the process of becoming ill as an oncological patient under palliative care in the home context. Method: this is a qualitative, exploratory and descriptive study, which was developed with ten oncological patients under palliative care, from May to July 2015. Moscovici's Social Representations Theory and thematic analysis were used. Results: the process of becoming ill brings losses and restrictions in what concerns daily life and earning capacity. In order to cope with it, the support network comes from family, social network, spirituality, and health providers. There has been some modifications in what concerns social representations that associate cancer to death, with treatment evolution and improvement of cancer prognosis. Therefore, the participants felt hopeful about cure. Final considerations: It was observed that being sick with cancer in palliative care can influence negatively due to restrictions in daily life and in productive capacity; In addition to modifying the social representation that associates cancer with death and, regardless of the stage of the patients presence, they presented hope of healing, associated or not with the faith.

Descriptors: Chronic disease; Neoplasms; Palliative care; Terminally ill.

RESUMEN: Objetivo: conocer las representaciones sociales del proceso de adolecimiento de pacientes oncológicos, en cuidados paliativos, en el contexto domiciliar. Método: estudio cualitativo, exploratorio y descriptivo, desarrollado con diez pacientes oncológicos en cuidados paliativos, entre mayo y julio de 2015, basado en el análisis temático y la Teoría de Representaciones Sociales, de Mocovici. Resultados: el proceso de enfermedad presenta pierdas, restricciones en la vida diaria y en la capacidad laboral. Para este enfrentamiento la red de apoyo se hace presente en la figura de la familia, de la red social, de la espiritualidad y del equipo de salud. Con la evolución del tratamiento y la mejora del pronóstico del cáncer, hubo modificaciones en la representación social que relaciona el cáncer con la muerte, así los participantes presentaron la esperanza de curarse. Consideraciones finales: Se observó que el adolecer de cáncer, en cuidados paliativos, puede causar restricciones en la vida diaria y en la capacidad productiva, además de modificar la representación social, la cual asocia el cáncer con la muerte, pues, independientemente de la etapa en la que los pacientes se encuentran, éstos presentaron esperanza de la curación, asociado o no la fe.

Descriptores: Enfermedad crónica; Neoplasias; Cuidados paliativos; Enfermo terminal.

\section{INTRODUÇÃO}

O câncer, atualmente, pode ser considerado um dos problemas mais complexos enfrentado no sistema de saúde pública, considerando sua importância epidemiológica, social e econômica. Ele constitui o nome dado a um conjunto de mais de 100 doenças, que têm em comum o crescimento desordenado de células que tendem a invadir tecidos e órgãos vizinhos. Considera-se que, pelo menos, um terço dos casos que ocorre anualmente no mundo poderia ser prevenido por intermédio da adoção de um estilo de vida saudável, evitando a exposição a substâncias que aumentem o risco de desenvolvimento do câncer. ${ }^{1}$ 


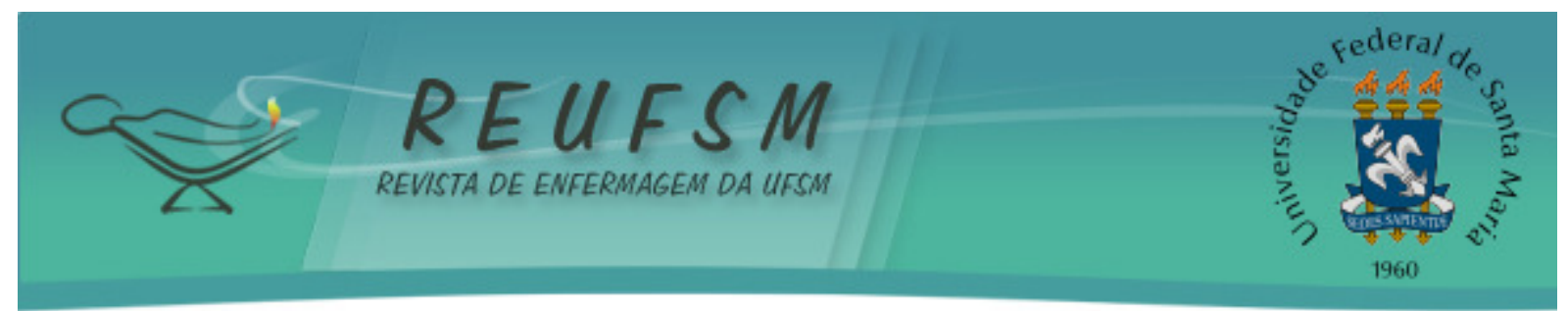

No Brasil, a estimativa para o biênio 2016-2017, aponta a ocorrência de cerca de 600 mil casos novos dessa doença. O perfil epidemiológico identifica que os cânceres de próstata (61 mil) em homens e mama (58 mil) em mulheres serão os mais frequentes no país. Sem contar os casos de pele não melanoma, os tipos mais frequentes em homens serão próstata $(28,6 \%)$, pulmão $(8,1 \%)$, intestino $(7,8 \%)$, estômago $(6,0 \%)$ e cavidade oral $(5,2 \%)$. E nas mulheres, os de mama $(28,1 \%)$, intestino $(8,6 \%)$, colo do útero $(7,9 \%)$, pulmão $(5,3 \%)$ e estômago $(3,7 \%)$ estão entre os principais. ${ }^{2}$

Os cuidados paliativos visam promover qualidade de vida, prevenindo e aliviando o sofrimento de indivíduos e de suas famílias na vigência de doenças crônicas não transmissíveis (DCNT) como o câncer e crescem em significado com a progressão da doença e o tratamento sem perspectiva de cura, com ameaça à vida. Entretanto, o ideal seria a paliação desde o momento do diagnóstico da doença, como estabelecido em seu conceito, a fim de se obter uma melhor qualidade de vida, sem sofrimento, com foco na pessoa e não mais na doença. ${ }^{3}$

No Brasil, as discussões a respeito dos cuidados paliativos são observadas desde os anos 70. Porém, os primeiros serviços começaram a surgir nos anos 90. Destaca-se a abertura dos primeiros cursos com filosofia paliativista na Escola Paulista de Medicina - UNIFESP/EPM pelo professor Marco Túlio de Assis Figueiredo. O Instituto Nacional do Câncer - INCA, também pioneiro, inaugurou em 1998 o hospital Unidade IV, exclusivamente dedicado aos Cuidados Paliativos. Em 2002, o Hospital do Servidor Público Estadual de São Paulo - HSPE/SP inaugurou sua enfermaria de cuidados paliativos, conduzida pela doutora Maria Goretti Sales Maciel. ${ }^{4}$

Contudo, a falta de informação de profissionais de saúde, bem como a não inserção na grade curricular de temas associados a finitude da vida ou cuidados paliativos, dificultam a qualificação profissional nesta área, o que favorece a paliação tardia e não compreensão de sua importância pelo paciente e família. ${ }^{3}$

Os princípios dos cuidados paliativos são: promover o alívio da dor e de outros sintomas desagradáveis; afirmar a vida e considerar a morte um processo normal, sem acelera-la ou adia-la; integrar os aspectos psicológicos e espirituais no cuidado; oferecer suporte possibilitando atividades até o momento da morte; conceder apoio para os familiares durante a doença do paciente e o luto; possibilitar abordagem multiprofissional para pacientes e familiares incluindo acompanhamento no luto; melhorar a qualidade de vida e influenciar positivamente o curso da doença; iniciar o mais precocemente possível o cuidado paliativo, 


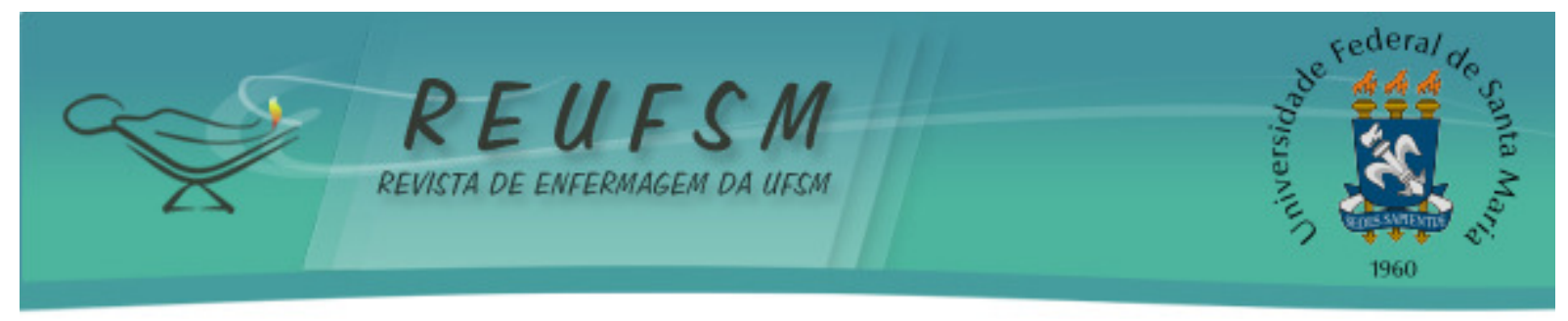

juntamente com outras medidas de prolongamento da vida, como quimioterapia e radioterapia, e incluir todas as investigações necessárias para melhor compreender e controlar situações clínicas estressantes. ${ }^{5}$

Foca-se o conceito de cuidado paliativo no contexto domiciliar, uma vez que o apoio familiar durante a atenção prestada à pessoa adoecida e a possibilidade da morte no domicílio configuram-se como pontos fundamentais. A provisão desse tipo de assistência realizada por serviços, como o do presente estudo, no ambiente domiciliar ainda é insuficiente comparada à necessidade e importância de cuidados paliativos nesse local. Neste sentido, a equipe de saúde, assume papel de destaque no que tange as orientações prestadas as pessoas atendidas e sua família tendo como foco assegurar esses cuidados com alta qualidade. ${ }^{6-7}$

Na tentativa de associar os cuidados paliativos por câncer ao referencial de Moscovici, temse como conceito que as pessoas e grupos criam representações sociais por meio da comunicação e, uma vez criadas, elas adquirem vida própria, circulam, se encontram, se atraem e se repelem, dando continuidade ao surgimento de novas representações, enquanto velhas representações morrem. ${ }^{8}$

Salienta-se ainda que o meio social em que a pessoa está inserida terá influência decisiva nas imagens que ela constrói e na forma como ela vivenciará este processo. Todo ambiente é composto por essas imagens que constantemente são acrescidas de algo e modificadas, descartam-se algumas e adotam-se outras. ${ }^{8}$

Assim, entende-se a importância de associar a Teoria das Representações sociais como referencial teórico. Nessa perspectiva, a questão de pesquisa foi: Quais as representações sociais do processo de adoecimento de pacientes oncológicos em cuidados paliativos? Tendo como objetivo conhecer as representações sociais do processo de adoecimento de pacientes oncológicos em cuidados paliativos no contexto domiciliar.

\section{MÉTODO}

Esta pesquisa se caracteriza como exploratória, descritiva, com abordagem qualitativa. ${ }^{9}$ Foi realizada com pacientes internados no Programa de Internação Domiciliar Interdisciplinar (PIDI) de um hospital de ensino do sul do Brasil. As internações no PIDI são direcionadas às pessoas com diagnóstico de câncer e que estão em cuidados paliativos, as quais são visitadas diariamente em suas residências pelas equipes de saúde. 


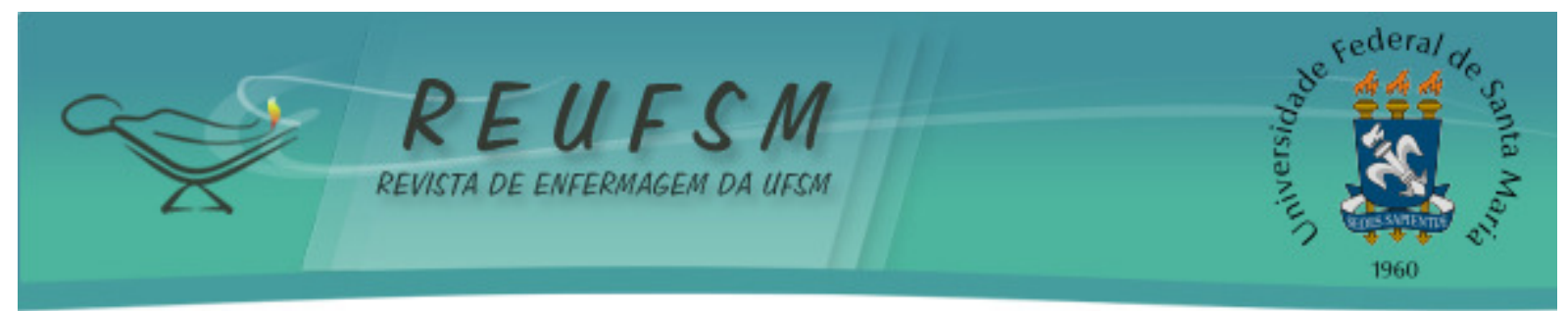

Os critérios de inclusão foram: idade superior ou igual a 18 anos; ter diagnóstico de câncer; estar internado no Programa de Internação Domiciliar em cuidados paliativos; comunicarse verbalmente e consentir com o uso do gravador durante as entrevistas. Apresentar condições clínicas incompatíveis com a realização de uma entrevista foi o critério de exclusão utilizado.

A coleta de dados ocorreu no período de maio a julho de 2015, por meio de entrevista narrativa. ${ }^{10}$ No momento inicial da coleta de dados haviam 16 pacientes em internação domiciliar, sendo que alguns pacientes foram a óbito e outros evoluíram para condições clínicas incompatíveis para suportarem uma entrevista. Assim, fizeram parte da pesquisa 10 pacientes. As entrevistas tiveram duração média de 30 minutos e as conversas informais, que foram anotadas em um diário de campo, em média 15 minutos.

As questões orientadoras da entrevista se relacionavam a vida, a presença/ausência de sinais e sintomas e as preocupações do paciente no contexto dos cuidados paliativos relacionados a doença oncológica.

O texto foi colocado em três colunas, a primeira englobava a transcrição, a segunda equivalia a primeira redução, e a terceira tinha palavras-chave. Desenvolveram-se então as categorias. Primeiramente, foram criadas categorias para cada entrevista narrativa, em seguida, foram ordenadas em um sistema de categorização geral para todas as entrevistas segundo a análise temática. ${ }^{10} \mathrm{O}$ produto final foi constituído da interpretação das entrevistas a partir da Teoria das Representações Sociais, reunindo as estruturas de relevância dos participantes com as do pesquisador.

O Projeto foi apreciado pelo Comitê de Ética em Pesquisa, tendo aprovação em maio de 2015, sob o número de Parecer 1.054.565 e CAAE 43887715900005316. Foram respeitados os princípios éticos estabelecidos pela Resolução 466/12. Para manter o anonimato, os participantes foram identificados pela letra $\mathrm{P}$ (participante), seguida por numeração em ordem crescente (P1, P2, P3, P4...).

\section{RESULTADOS E DISCUSSÃO}

O câncer é uma doença silenciosa que somente é percebida pela pessoa quando do surgimento dos sinais e sintomas que prejudicam as suas atividades diárias e laborais. $\mathrm{O}$ fato de apresentar alguma doença, geralmente, traz consigo algumas limitações nas atividades antes realizadas, principalmente, relacionadas à capacidade para o trabalho. Nesse sentido, 


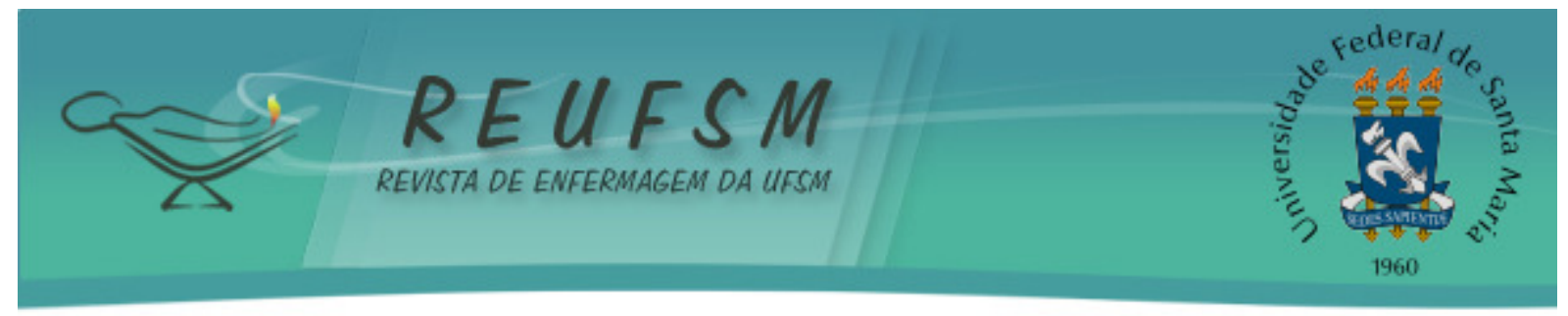

esta condição foi vista como uma grande perda para os pacientes, uma vez que trabalhar estava diretamente relacionado à certeza de sua capacidade e integridade física, independência, realização pessoal, autoestima e estabilidade financeira. Nas narrativas a seguir identificou-se que, com o aparecimento ou diagnóstico da doença, as atividades de trabalho foram interrompidas.

Na lavoura eu não parava nunca e tinha os bichos na volta da casa para dar "boia" [comida] [...]. (P8)

\section{Eu fazia tudo antes, não parava nunca. (P3)}

É a partir deste contexto, por meio da ancoragem e da objetivação que as representações sociais sobre doença são construídas. ${ }^{11}$ Objetivar é reproduzir um conceito em uma imagem, assim as imagens selecionadas passam a ser integradas no que é conhecido como núcleo figurativo. ${ }^{8}$ Nesse sentido, o estar doente representou diminuição da produtividade.

Corroborando com esses dados, um estudo que visou conhecer as representações sociais de homens sobre saúde e doença, identificou que, quando indagados sobre a definição de doença, esta é compreendida como algo que tende a romper ou prejudicar a realização de atividades da rotina diária, principalmente relacionadas ao trabalho, e que isso perpassa a experiência do adoecimento. ${ }^{12} \mathrm{O}$ paciente, que antes vivenciava uma vida "normal", produtiva, gradualmente perde muitas funções, percebendo-se diante de limitações. ${ }^{13}$

Estas restrições vivenciadas pelos pacientes estavam entrelaçadas aos sinais e sintomas que acompanhavam o processo de adoecer por câncer. A fadiga foi um dos sintomas destacados pelos participantes deste estudo.

Quando eu vou caminhar eu sinto peso nas pernas, me canso e me dá um tipo de uma ânsia [...] estou sentindo que estão enfraquecendo, sinto peso, mas toda hora eu estou fazendo exercício, eu nunca deixo parar. (P5)

Eu fico fraca, [...] quando eu falo demais. (P9)

A gente fica prostrado assim, mais desanimado. (P3)

As narrativas revelaram que a fadiga é um sintoma que traz consigo muitas impossibilidades relacionadas às atividades da rotina diária e ao autocuidado, caracterizando a doença como impositiva de atividades restritas a não utilização de muita energia. A fisiopatologia da fadiga relacionada ao câncer é pouco conhecida, mas várias causas podem sobrepor-se e contribuir para o seu agravamento. É, muitas vezes, descrita como o primeiro sinal de problemas de saúde, sendo identificada como angustiante porque se opõe às 


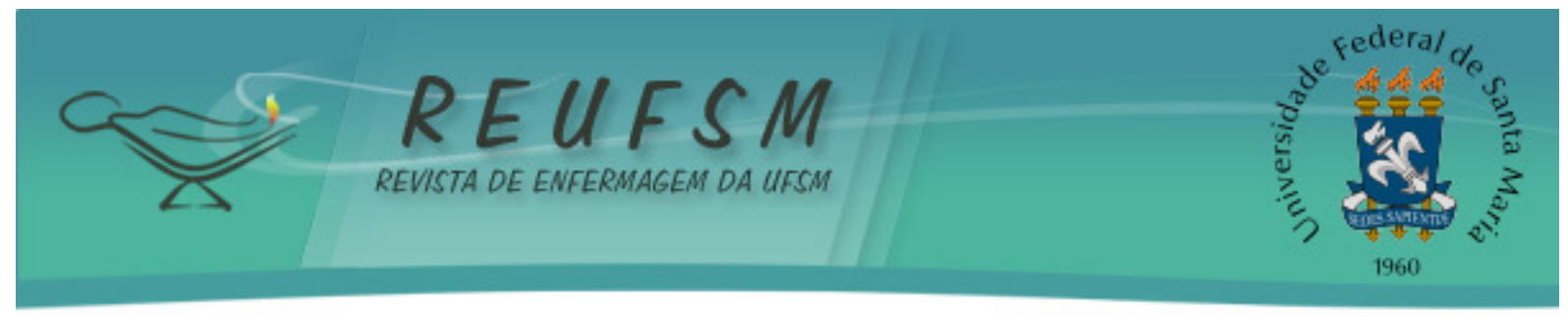

atividades rotineiras, laborais, de afazeres e de lazer normais do indivíduo, causando frustração, tristeza, aflição e angústia. ${ }^{14}$

Ainda, foram destacados pelos participantes do estudo os sintomas ligados ao aparelho digestivo, os quais representavam fatores negativos no viver do paciente oncológico em cuidados paliativos.

Eu notei que comecei a emagrecer, a perder o apetite, aí já vi que tinha alguma coisa errada. (P7)

Tinha dificuldade para engolir, fui me enfraquecendo. (P10)

E me deu um desarranjo [diarreia] e não parou mais, e eu já comecei a não comer mais nada. (P1)

A inapetência, a disfagia e a diarreia foram sintomas preocupantes que estiveram presentes no início da doença e que indicaram que algo estava errado, sendo um dos principais motivos para procura de um serviço de saúde. Identificou-se assim que estar doente e em cuidados paliativos também tem como forte representação social ter dificuldades quanto à alimentação.

Ainda, constatou-se que os sintomas digestivos podem estar diretamente relacionados ao desenvolvimento do câncer ou podem surgir em decorrência do tratamento quimioterápico, como um dos efeitos adversos desta terapêutica.

Depois que eu faço [quimioterapia] eu passo bem, eu vomito uma vez por dia só, mas nas vésperas sim, dois dias antes de fazer de novo aí eu vomito bastante, um enjoamento, coisa mais séria! (P8)

No primeiro dia que eu fiz [quimioterapia], não sei se estava muito lá em baixo, me deu uma semana de diarreia, fiquei ruim [...] me dava dois, três dias de náusea. (P3)

Eu perdia a fome com esse remédio [quimioterapia], eu estava muito magro, eu não almoçava e desmaiei na sala dela [médica]. (P2)

As narrativas trouxeram as experiências dos participantes P8, P3 e P2 quanto aos efeitos colaterais da quimioterapia em momentos pregressos, mas também do período em que ocorreram as entrevistas, com enfoque paliativo. Sabe-se que o controle dos sintomas é um dos principais objetivos dos cuidados paliativos, pois estarão diretamente relacionados à qualidade de vida. A presença destes colabora para a perpetuação das representações sociais negativas relacionadas ao câncer, tais como sofrimento, doença incapacitante, pavor e medo.

Em um estudo desenvolvido no referido serviço de atenção domiciliar, que objetivou conhecer aspectos relacionados ao processo de morte e morrer, foi ressaltado que os pacientes que recebiam essa atenção manifestavam momentos em que estavam melhores diante da 


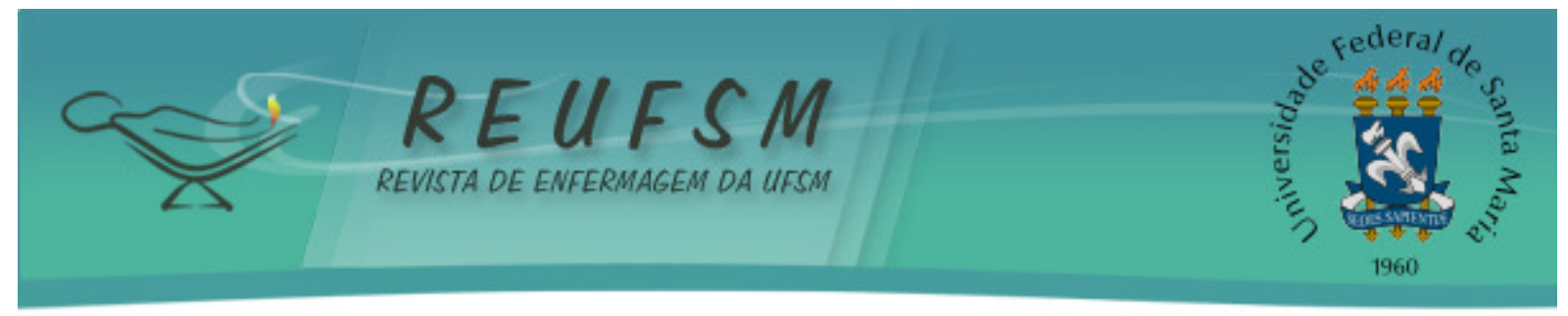

ausência dos sintomas físicos, entretanto, quando eles persistiam como a dor e as náuseas, o viver tornava-se mais difícil. ${ }^{15}$

A quimioterapia é uma forma de tratamento sistêmico do câncer que utiliza medicamentos administrados continuamente ou em intervalos regulares, variando conforme os esquemas terapêuticos específicos para cada tumor e estadiamento da doença. ${ }^{2}$ Esse tratamento inclui o foco paliativo que é indicado para paliar sinais e sintomas que podem prejudicar a capacidade funcional da pessoa com a doença, entretanto, não influencia necessariamente na sobrevida. ${ }^{1}$

Independente do seu objetivo, paliativo ou não, a quimioterapia pode ocasionar diversos efeitos colaterais, inclusive relacionados ao aparelho digestivo e que estão associados a este tratamento, tais como xerostomia, mucosite, inapetência, náuseas, vômitos, perda de peso, diarreia e constipação. Todos estes sinais e sintomas produzem um impacto significativo na vida dos pacientes. ${ }^{16}$

Os participantes do estudo narraram a respeito de experiências que marcaram o viver com a doença oncológica, ressaltando outro efeito do tratamento quimioterápico: à alopecia, sinal característico da pessoa com câncer.

Outra coisa também que foi bem difícil, foi quando eu tive que explicar para ele [filho] que eu ia perder o cabelo, foi mais dificil. (P3)

O enfermeiro me colocou um líquido vermelho e disse assim: "esse é de cair o cabelo.". Mas quem sabe, que eu tenha um pouco de sorte que não caia, tem tanta gente que tem os cabelinhos. (P9)

A alopecia está diretamente associada ao câncer, sendo uma das marcas de sua identificação e pode representar para o paciente e para a sua família a certeza de que a doença é mesmo um câncer. ${ }^{17}$ Esse sinal induzido pela quimioterapia é classificado como um dos três principais efeitos colaterais mais angustiante por pacientes e pode causar baixa qualidade de vida, níveis elevados de angústia e alterações na autoimagem. ${ }^{18}$ Desse modo, apareceu como representação social negativa associada ao estar doente por câncer.

Percebeu-se então, que o processo frente ao adoecer por câncer, doença ameaçadora da vida, e os tratamentos que foram implantados trouxeram modificações na vida destas pessoas e a atuação dos profissionais de saúde frente a cada um desses momentos pode modificar este viver.

É nesta perspectiva que a vertente paliativa tem um sentido relevante, estando esses cuidados recomendados as pessoas que têm uma doença que coloca em risco a sua vida, mas 


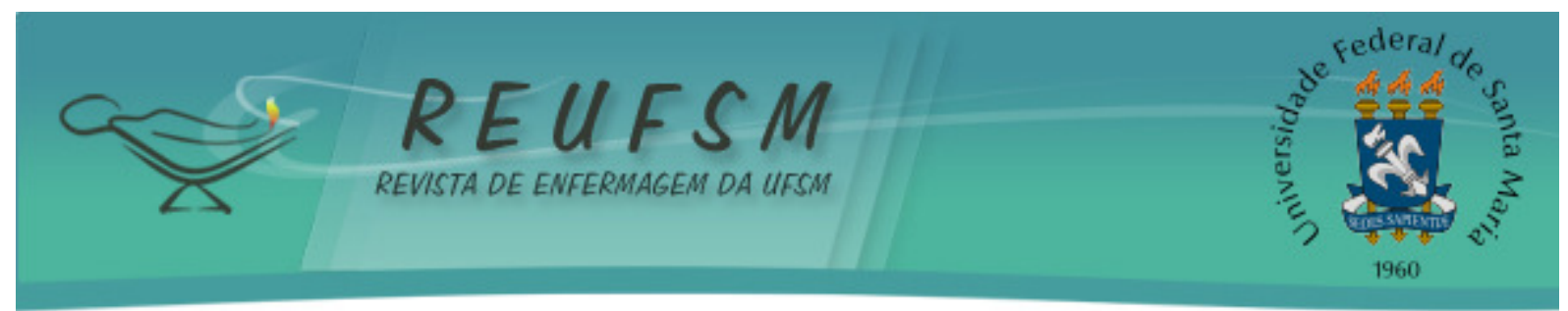

também atenção a seus familiares, independente do diagnóstico e prognóstico, da idade, sobretudo, em qualquer etapa da doença, mas que necessite de cuidados para atender suas necessidades. ${ }^{19}$

Sendo assim, percebe-se que os cuidados paliativos têm justamente este foco, o cuidado. Um cuidado que visa propiciar, por meio do alívio dos sintomas, a melhoria da qualidade de vida da pessoa que está diante de um impacto social, emocional, psicológico, físico e espiritual imposto pela doença. ${ }^{20}$

Nesse contexto, a rede de apoio foi importante, com vistas a auxiliar no processo de enfrentamento da doença. Os participantes destacaram a sua rede de apoio conforme exposto a seguir.

No começo foi difícil [diagnóstico da doença], eu, se não fosse a minha família, a fé que a gente tem, eu não sei o que tinha acontecido. (P3)

O meu namorado deu bastante força, ele, a família toda [...], Os médicos foram me tranquilizando também, a minha fé me ajudou bastante. (P7)

Eu tenho fé em Deus. (P8)

Depois que eu sou testemunha de Jeová, eu me sinto muito feliz, eu sinto a ajuda dele [...] a minha família todos me ajudam [...] muitas amigas, pessoas até de outras cidades têm vindo aqui me ver [...] até o Tobinho [cachorro] me ama. (P6)

As ações de cuidado como a ajuda, o apoio, a companhia, além de sentimentos como afeto, apreço e amor, tanto de familiares, quanto de amigos, vizinhos e pessoas de sua convivência, resultam em relações de confiança e fidelidade, fazendo com que os pacientes se sintam mais seguros e protegidos. ${ }^{21}$ A rede de apoio tem um papel importante para o paciente com câncer devido sua relação horizontal e de aproximação envoltas de sentimentos como o amor, a solidariedade e a gratidão. ${ }^{22}$

No cenário dos cuidados paliativos do paciente oncológico é evidenciado que o apoio da família é primordial para o enfrentamento da doença e superação dos percalços. Ainda, é descrito que as crenças espirituais e religiosas contribuem para o enfrentamento do processo de adoecimento. ${ }^{15}$

O contexto do cuidado domiciliar também foi destacado pelos pacientes.

Quando que eu ia poder me tratar do jeito que o PIDI me trata, médico, enfermeiro, nutricionista, chegou a ter oito aqui para me ver em uma manhãa, então para mim é muito bom, então para mim está tudo bem. (P2) 


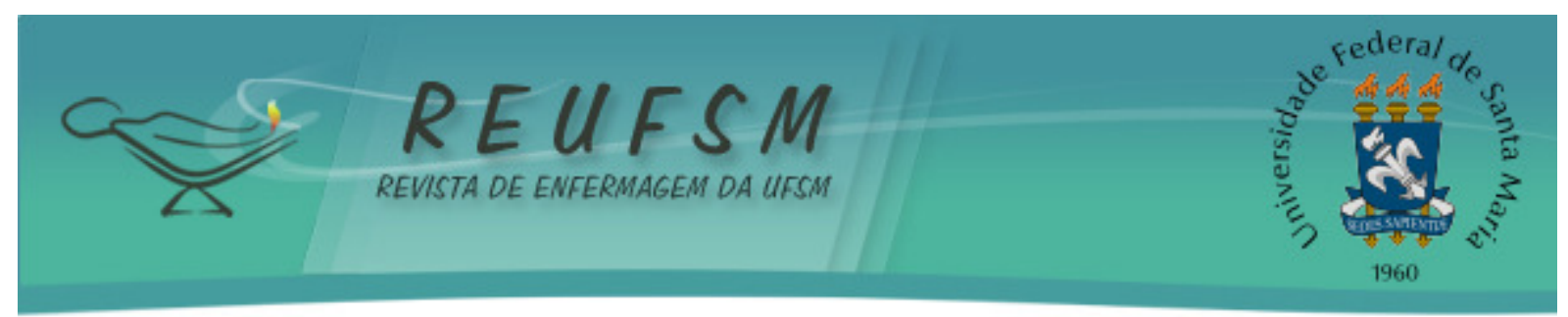

Estou me sentindo muito bem! Muito bem tratado, pessoal muito bem treinado, muito educado [...] o importante é que as pessoas sejam bem tratadas, com carinho, com amor. (P4)

O ambiente domiciliar também constitui cenário de cuidado, pelos benefícios que permite ao paciente e sua família, pois é um espaço que possibilita que o cuidado esteja mais presente, tanto por parte da família quanto por parte dos profissionais de saúde, onde os vínculos são fortalecidos e a rotina da vida diária pode ser mantida.

Deste modo, é relevante que os profissionais de saúde desenvolvam um relacionamento interpessoal positivo com paciente e família mediante a comunicação, a fim de promover uma assistência humanizada, holística e de qualidade ao paciente sem possibilidade de cura. ${ }^{23}$ Ainda, destaca-se que os cuidados paliativos proporcionados pelos profissionais da enfermagem tem em sua essência a atenção as dimensões físicas, psicológicas, sociais e espirituais, sobretudo, diante do processo de morrer na ambiente domiciliar. ${ }^{24}$

Os profissionais da saúde também representam uma fonte de apoio, sobretudo, uma equipe assistencial que atenda no domicílio da pessoa com a doença e que promova um cuidado pautado na humanização, visando atender as necessidades biopsicossociais e espirituais. ${ }^{15}$

Considerando que as representações sociais ingressam no mundo comum e cotidiano vivenciado e discutido com os amigos e colegas e que circulam na mídia diariamente. ${ }^{6}$ Destaca-se que a internação domiciliar como modalidade de atenção neste estudo apresentouse ligada às representações sociais positivas, como proteção, segurança e apoio de uma equipe de saúde de referência.

Observou-se também, que a esperança da cura foi fortemente identificada nos depoimentos dos participantes.

Eu estou tranquila, eu sei que Deus é maior, se eu tiver que me salvar eu vou me salvar. Vou me salvar se Deus quiser, eu confio nele. (P9)

Só estou louco para melhorar de uma vez, para retirar essa sonda. (P10)

Fui para Porto Alegre para ver se tinha alguma solução, procurar mais ajuda. (P5)

Eu sinto que estou me curando, graças a Deus acho que falta pouco. (P2)

Eu tenho certeza de que vou ficar boa, que eu vou ficar curada, sabe. (P7)

Neste contexto, a esperança da cura foi percebida, ocorrendo como uma defesa, negação do processo de adoecimento, pois os pacientes atendidos pelo serviço selecionado foram comunicados sobre suas condições clínicas e possibilidades de cura durante o tratamento e 


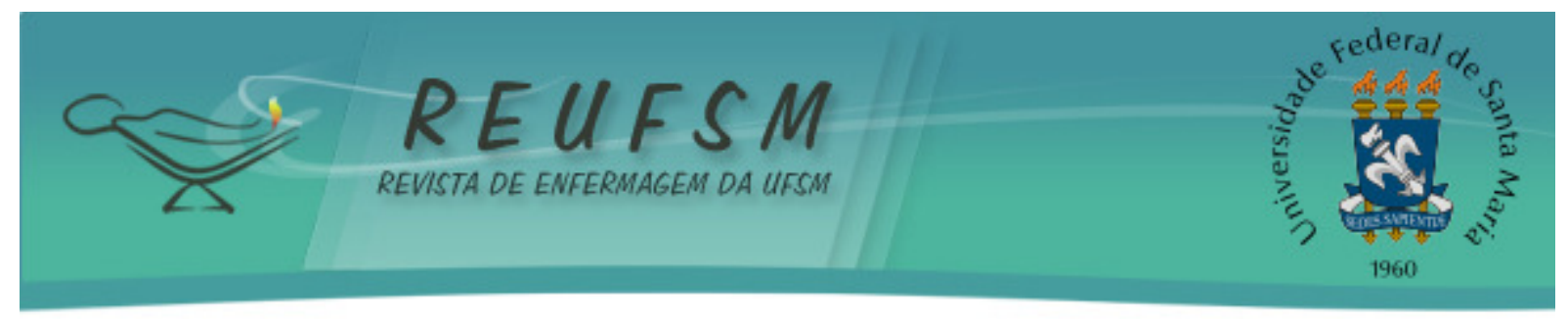

acompanhamento. A esperança permanece durante todo o processo de adoecimento até a morte por possibilitar apoio psicológico aos pacientes, por dar uma sensação de poder suportar por mais algum tempo e assim conseguir animo para superarem as diversidades do tratamento. ${ }^{15}$

Outrossim, na perspectiva da esperança de vencer o câncer, este sentimento pode estar ancorado na fé ou religiosidade. Deus pode ser considerado o depósito das expectativas de cura ou representar o motivo de vivenciar um câncer sem perspectiva de cura, ou ainda ser considerado o "ser supremo" é o único responsável pelo destino de todos, ou seja, neste sentido conseguem lidar com a situação de final de vida, encontrando em Deus ou na religião este suporte. ${ }^{25}$

\section{CONSIDERAÇÕES FINAIS}

A partir do estudo pode-se identificar que o câncer apresenta como núcleo figurativo a diminuição da capacidade produtiva, a presença do cansaço e as dificuldades relacionadas à alimentação. Destaca-se a relevância do trabalho e da alimentação como representações do viver dos seres humanos, portanto, a influência negativa da dificuldade e impossibilidade dessas atividades no curso do adoecimento por câncer é ressaltada.

Percebeu-se que, independente do estágio em que o paciente se encontrasse, a esperança da cura se mantém presente, podendo ter relação com religiosidade ou por negação à doença, não havendo assim modificações em relação à representação social que associa o câncer à morte.

Reitera-se a importância da equipe de saúde durante todo esse processo, a qual deve ser sensível a este viver, disposta a escutar, a acolher as dúvidas e as incertezas desta caminhada, que não são poucas. Ainda necessita orientar quanto aos cuidados e estratégias existentes para minimizar os efeitos colaterais decorrentes do tratamento e que se mostre parceira neste. Assim, proporcionar-se-á que esta vivência se transforme e que as representações sociais construídas a partir de então também sejam modificadas positivamente.

Ademais, salienta-se que a atenção domiciliar constitui uma importante unidade de cuidado, visto que aproxima as ações assistenciais do ambiente vivido pelo paciente e sua família, o que repercute em um cuidado humanizado e de acordo com as necessidades biopsicossociais e espirituais das pessoas atendidas, sobretudo, pela aproximação com o contexto de vida delas.

Entende-se que a limitação do estudo, o reduzido número de participantes, estava diretamente relacionada com a população escolhida: pessoas com câncer em cuidados paliativos, as quais eram atendidas em um único serviço do município. Todavia, o objetivo foi 


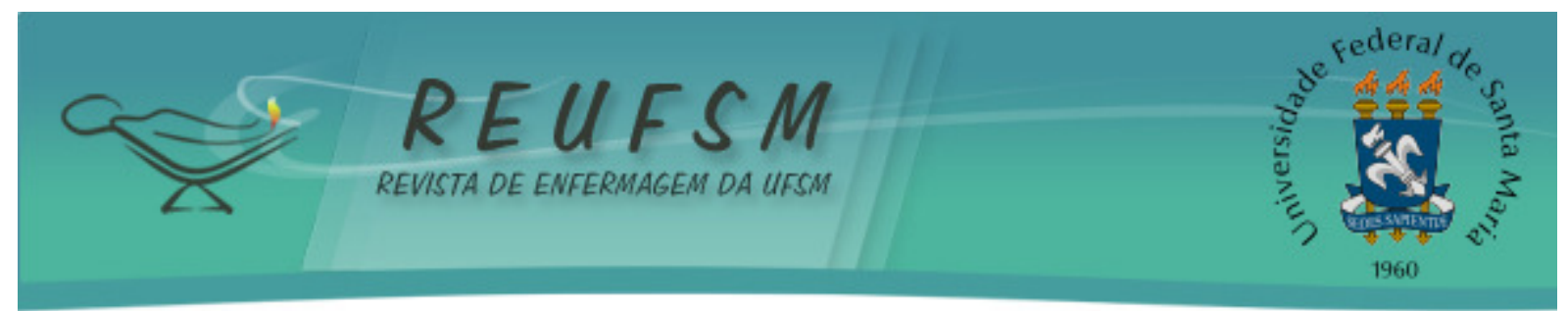

atingido, pois as representações sociais do processo de adoecimento dos pacientes oncológicos em cuidados paliativos foram conhecidas. Sugere-se a realização de pesquisas que visem interferir no contexto das pessoas com câncer, e que, portanto, venham colaborar e influenciar positivamente nos percalços encontrados pelos pacientes oncológicos.

\section{REFERÊNCIAS}

1. Instituto Nacional de Câncer (BR). ABC do câncer: abordagens básicas para o controle do câncer. Rio de Janeiro (RJ): Inca; 2012.

2. Instituto Nacional de Câncer (BR). Estimativa 2016: Incidência de Câncer no Brasil. Rio de Janeiro (RJ): Inca; 2015.

3. Rodrigues LA, Ligeiro C, Silva M. Cuidados paliativos, diagnósticos e terminalidade: indicação e início do processo de paliação. Rev Cuidarte Enf. 2015;9(1):26-35.

4. Academia Nacional de Cuidados Paliativos. [internet] 2017 [acesso em 2017 ago 30]. Disponível em: http://paliativo.org.br/cuidados-paliativos/cuidados-paliativos-no-brasil/

5. WHO. Definition of Palliative Care. [internet] 2010 [acesso em 2017 jul 14] Disponível em: http://www.who.int/cancer/palliative/definition/en/

6. Robinson CA, Bottorff JL, McFee E, Bissell LJ, Fyles G. Caring at home until death: enabled determination. Supportive Care in Cancer. 2017;25(4):1229-1236.

7. Morin L, Aubry R. Palliative care in hospital-at-home services in France/Soins palliatifs en hospitalisation à domicile: A nationwide study using administrative data/état des lieux à partir des données hospitalières. Médecine palliative. 2017;16(1):7-20.

8. Moscovici S. Representações Sociais: investigações em psicologia. Petrópolis: Vozes; 2003.

9. Minayo MCS. O desafio do conhecimento: pesquisa qualitativa em saúde. $14^{a}$ ed. São Paulo: HUCITEC; 2014.

10. Jovchelovitch S, Bauer MW. Entrevista narrativa. In: Bauer MW; Gaskell G (organizadores). Pesquisa qualitativa com texto, imagem e som: um manual prático. $11^{\mathrm{a}}$ ed. Rio de Janeiro: Vozes; 2013.

11. Jovchelovitch S. Vivendo a vida com os outros: intersubjetividade, espaço público e representações sociais. In: GUARESCH, P.; JOVCHELOVITCH, S. (Organizadores). Textos em representações sociais. 14 ${ }^{\text {a }}$ ed. Rio de Janeiro: Vozes; 2013.

12. Hemmi APA, Conceição JA, Santos DDM. Representações sociais de homens sobre saúde e doença: contribuições para o cuidado. Rev Enferm Cent-Oest Min. 2015;5(1);1457-68.

13. Oliveira DC. Construção e transformação das representações sociais da Aids e implicações para os cuidados de saúde. Rev latinoam enferm [internet] 2013 [acesso em 2015 out 5];21(Spec):276-86. Disponível em: http://www.scielo.br/pdf/rlae/v21nspe/pt_34.pdf

14. Chiba T, Cabral LRB. Fadiga, sudorese e prurido. In: Academia Nacional de Cuidados Paliativos (organizadores). Manual de Cuidados Paliativos ANCP. $2^{\text {a }}$.ed. Porto Alegre: Solo editoração e design gráfico LTDA; 2012. 


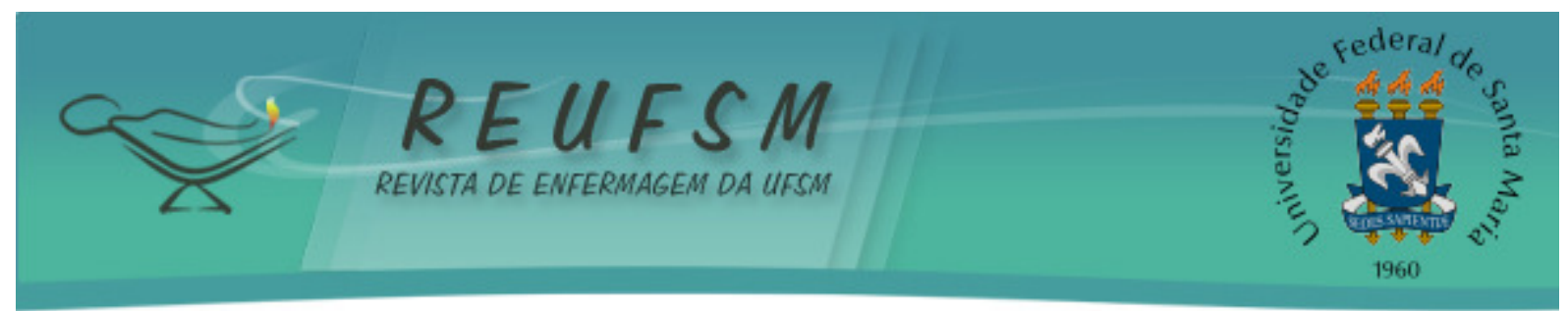

15. Tomaszewski AS, Oliveira SG, Arrieira ICO, Cardoso DH, Sartor SF. Manifestações e necessidades referentes ao processo de morte e morrer: perspectiva da pessoa com câncer. Rev Fund Care on line. [internet] 2017 [acesso em 2017 jul 13];9(3):705-16. Disponível em:

http://seer.unirio.br/index.php/cuidadofundamental/article/view/5503

16. Silva JMC, Ribeiro PPSV. Estratégias de autocuidado das pessoas com doença oncológica submetidas a quimioterapia/radioterapia e a sua relação com o conforto. Enferm glob. 2015;14(1):372-400.

17. Otani MAP, Barros NF, Marin MJS. A experiência do câncer de mama: percepções e sentimentos de mulheres. Rev baiana enferm. 2015;29(3):229-39.

18. Shaw J, Boyle F. Chemotherapy induced alopecia and strategies to manage its impact. Cancer forum. 2017; 41(1): 40-45.

19. Sociedade Brasileira de Geriatria e Gerontologia (BR). Vamos falar de cuidados paliativos. [internet] 2015 [acesso em 2017 jul 13] Disponível em: http://sbgg.org.br/wpcontent/uploads/2014/11/vamos-falar-de-cuidados-paliativos-vers--o-online.pdf

20. Vasconcelos EV, Santana ME, Silva SED. Desafios da enfermagem nos cuidados paliativos: revisão integrativa. Enferm foco. 2012;3(3):127-30.

21. Carvalho SORM, Budó MLD, Silva MM, Alberti GF, Simon BS. "Com um pouco de cuidado a gente vai em frente": vivências de pessoas com estomia. Texto \& contexto enferm. 2015;24(1):279-87.

22. Lerner K, Vaz P. "My story of overcoming": suffering, testimony and therapeutic practices in cancer narratives. Interface comun saúde educ. 2017;21(61):153-63.

23. Andrade CG, Costa SFG, Costa ICP, Santos KFO, Brito FM. Cuidados paliativos e comunicação: estudo com profissionais de saúde do serviço de atenção domiciliar. Rev pesqui cuid fundam (online). [internet] 2017 [acesso em 2017 abr 5];9(1):215-221. Disponível em: http://www.seer.unirio.br/index.php/cuidadofundamental/article/view/5368/pdf_1

24. Sousa JM, Alves ED. Cuidados paliativos de enfermagem na atenção domiciliar. Rev enferm UFPE on line. [internet] 2015 [acesso em 2017 abr 2];9(2):669-76. Disponível em:

https://periodicos.ufpe.br/revistas/revistaenfermagem/article/view/10386/11137

25. Reis CGC, Farias CP, Quintana AM. O vazio de sentido: suporte da religiosidade para pacientes com câncer avançado. Psicologia: Ciência e Profissão. 2017;37(1): 106-18.

Data de submissão: 06/02/2017

Data de aceite: 12/09/2017

Autor correspondente: Michele Rodrigues Matos

E-mail: michele.rodriguesmatos@gmail.com

Endereço: Avenida Ferreira Viana, 3010, casa 11, bairro areal, Pelotas, RS.

CEP: $96085-000$ 